\title{
ERRATUM
}

\section{Erratum to: Arctic multiyear sea ice variability observed from satellites: a review*}

\author{
BI Haibo ${ }^{1,2,3,4, \#, * *}$, LIANG Yu 1,2,3,4, , WANG Yunhe ${ }^{1,2,3,4,{ }^{* *}}$, LIANG Xi ${ }^{5}$, ZHANG Zehua ${ }^{1,2,3,4}$, \\ DU Tingqin ${ }^{5}$, YU Qinglong ${ }^{6}$, HUANG Jue ${ }^{7}$, KONG Mei ${ }^{8}$, HUANG Haijun ${ }^{1,2,3,4}$ \\ ${ }^{1}$ CAS Key Laboratory of Marine Geology and Environment, Institute of Oceanology, Chinese Academy of Sciences, Qingdao \\ 266071, China \\ ${ }^{2}$ Laboratory for Marine Geology, Qingdao National Laboratory for Marine Science and Technology, Qingdao 266200, China \\ ${ }^{3}$ Center for Ocean Mega-Science, Chinese Academy of Sciences, Qingdao 266071, China \\ ${ }^{4}$ University of Chinese Academy of Sciences, Beijing 100049, China \\ ${ }^{5}$ Shandong Academy of Environmental Science Co., Ltd., Jinan 250013, China \\ ${ }^{6}$ National Marine Environmental Forecasting Center, Beijing 100081, China \\ ${ }^{7}$ Shandong University of Science and Technology, Qingdao 266590, China \\ ${ }^{8}$ Shandong Provincial Eco-environment Monitoring Center, Jinan 250101, China
}

(C) Chinese Society for Oceanology and Limnology, Science Press and Springer-Verlag GmbH Germany, part of Springer Nature 2021

\section{Erratum to: https://doi.org/10.1007/s00343-020-0093-7}

The affiliations of the authors of this article unfortunately contained a mistake. The corrected version is given below:

BI Haibo ${ }^{1,2,3,4, \#, * *}$, LIANG Yu1,2,3,4,\#, WANG Yunhe ${ }^{1,2,3,4, * *}$, LIANG Xi ${ }^{5}$, ZHANG Zehua ${ }^{1,2,3,4}$, DU Tingqin ${ }^{6}$, YU Qinglong ${ }^{5}$, HUANG Jue ${ }^{7}$, KONG Mei $^{8}$, HUANG Haijun ${ }^{1,2,3,4}$

\footnotetext{
${ }^{I}$ CAS Key Laboratory of Marine Geology and Environment, Institute of Oceanology, Chinese Academy of Sciences, Qingdao 266071, China

${ }^{2}$ Laboratory for Marine Geology, Qingdao National Laboratory for Marine Science and Technology, Qingdao 266200, China

${ }^{3}$ Center for Ocean Mega-Science, Chinese Academy of Sciences, Qingdao 266071, China

${ }^{4}$ University of Chinese Academy of Sciences, Beijing 100049, China

${ }^{5}$ National Marine Environmental Forecasting Center, Beijing 100081, China

${ }^{6}$ Shandong Academy of Environmental Science Co., Ltd., Jinan 250013, China

${ }^{7}$ Shandong University of Science and Technology, Qingdao 266590, China

${ }^{8}$ Shandong Provincial Eco-environment Monitoring Center, Jinan 250101, China
}

The online version of the original articles can be found at: https://doi.org/10.1007/s00343-020-0093-7.

\footnotetext{
* Supported by the National Key Research and Development Program of China (No. 2017YFC1404000), the National Natural Science Foundation of China (No. 41406215), the NSFC-Shandong Joint Fund for Marine Science Research Centers (No. U1606401), the Qingdao National Laboratory for Marine Science and Technology, the Postdoctoral Science Foundation of China (No. 014M561971), and the Open Funds for the Key Laboratory of Marine Geology and Environment, Institute of Oceanology, Chinese Academy of Sciences (No. MGE2020KG04)

** Corresponding authors: bhb@qdio.ac.cn; yhw1118@yeah.net

\# BI Haibo and LIANG Yu contributed equally to this work and should beregarded as co-first authors.
} 